\title{
Automatic determination of fiber orientation on OSB surface using line detection method based on small eigenvalue analysis
}

\author{
Chuanshuang Hu${ }^{\mathrm{a}}$, Chiaki TANAKA ${ }^{\mathrm{b} *}$, Reynolds OKAI ${ }^{\mathrm{c}}$, Tadashi OHTANI ${ }^{\mathrm{d}}$ \\ ${ }^{a}$ College of Forest, South China Agricultural University, Wushan, Tianhe District, Guangzhou, Guangdong, P.R. of China \\ $\mathrm{b}$ Department of Environmental Science and Technology, Kagoshima University, Kagoshima, Japan \\ ${ }^{\mathrm{c}}$ Department of Technology Education, University of Education, Winneba, Kumasi Campus, PO Box 1277, Kumasi, Ghana \\ ${ }^{d}$ Faculty of Science and Engineering, Shimane University, Matsue, Shimane, Japan
}

(Received 22 March 2004; accepted 22 February 2005)

\begin{abstract}
The modulus of rupture (MOR) which is the key to the strength of oriented strand board (OSB) depends on the fiber orientation on OSB surface. In order to improve the quality of OSB products, it is essential to optimize the flake alignments of the wood strands. In this paper, a line detection method based on small eigenvalue in conjunction with Canny edge detector was adopted to simulate the fiber strands with the ultimate aim of optimizing the fiber orientation and quality of the manufactured product. Then ellipse fitting analysis and/or line fitting analysis were used to determine the fiber orientation for all line or ellipse connectedness labeling, which expresses the fiber strands. The fiber orientation measured automatically was compared to that measured manually. It was observed that the ratio of the fiber orientation measured manually to that measured automatically was close to unity. The results indicated that the method developed in this paper when used to determine fiber orientation of the face surface in OSB was effective. However, the method developed in this paper was not effective for the under surface because small strands tend to fall when the face and middle layer were distributed, on the edge formation of fiber strands.
\end{abstract}

orientated strand boards / fiber orientation / image analysis

Résumé - Détermination automatique de l'orientation des fibres à la surface d'un panneau OSB au moyen d'une méthode de détection basée sur l'analyse des petites valeurs propres. Le module de rupture (MOR), facteur clé de la résistance des panneaux à lamelles orientées (OSB), dépend de l'orientation des fibres à la surface du panneau. Afin d'améliorer la qualité des produits OSB, il est essentiel d'optimiser l'arrangement des lamelles. Dans ce papier, une méthode de détection de contour basée sur l'analyse des petites valeurs propres, en association avec la méthode de détecteur de Canny, a été adoptée pour simuler les lamelles, avec comme objectif final d'optimiser l'orientation des fibres et la qualité du produit fini. Ensuite une analyse par identification d'une ligne ou d'une ellipse a été utilisée pour déterminer l'orientation des fibres pour toute connectivité identifiée, correspondant aux lamelles. L'orientation des fibres mesurée automatiquement a été comparée à celle mesurée manuellement. On a observé que le rapport entre les deux était proche de l'unité. Ces résultats montrent que la méthode développée dans cet article est efficace pour déterminer l'orientation des fibres sur la face d'un panneau OSB. Cependant, elle ne l'est pas pour déterminer l'orientation des fibres en dessous de la surface car de petites lamelles tendent à se placer sur les bords quand les couches extérieures et centrales ont des orientations distribuées dans leur plan.

panneau à lamelles orientées / orientation des fibres / analyse d'image

\section{INTRODUCTION}

Some wood species have received little attention in terms of usage because of their perceived poor physical and mechanical properties. An engineered wood has almost uniform physical and mechanical properties compared to conventional wood. Besides, there is a remarkable improvement in the mechanical properties of engineered wood products. Thus engineered wood products like OSB could promote the use of species with low mechanical properties. Now the use of OSB is gaining increasing popularity in Europe, North America and Asia. It has been established that the density of wood based panels has a direct impact on properties, such as stiffness and strength [5].
However, the amount and condition of the raw material in the mat, together with the pressing conditions, determine the average resultant density of wood based materials. It has also been established that density variations in both the horizontal and vertical planes of wood based panel products inevitably occurs. The vertical density distribution from a higher density at the surface to a lower density in the core is found to depend on the pressing conditions and extent of resin cure $[4,8]$. On the other hand, the horizontal density distribution is attributed to the mat formation process and layout of the wood strands [2].

OSB comprises large size element which are wood strand, and its mechanical properties depend on the fiber orientation of the strands such as MOR, which is the key to the strength of

* Corresponding author: tanakach@agri.kagoshima-u.ac.jp 


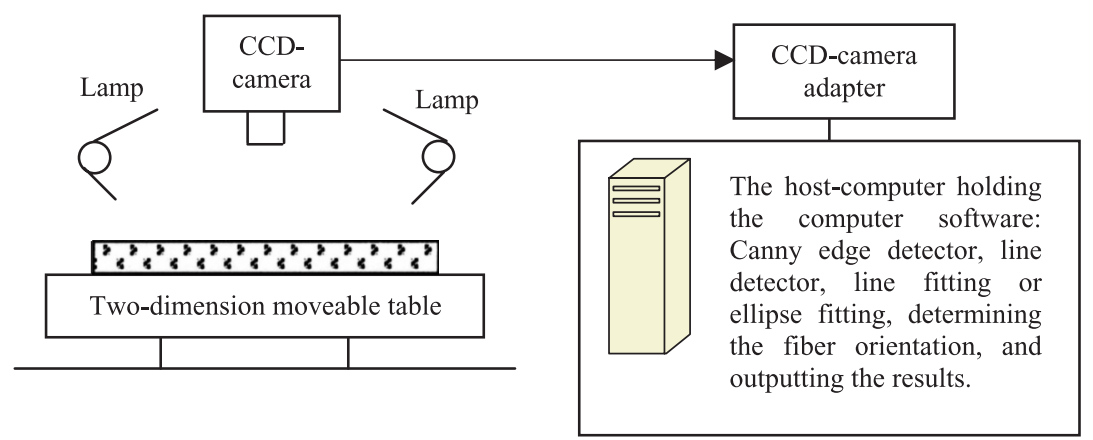

Figure 1. The schematic diagram of the system.

OSB in bending. In order to improve the quality of OSB products, it is essential to optimize the arrangement or fiber orientation of the wood strands. Pinto et al. (2003) have shown that image processing technology is useful in the application of wood products [7]. Nishimura et al. conducted studies on the relationship between the surface strands and MOR by applying the technique of image analysis (IA) [6]. In this paper, a novel image processing method based on line detection analysis was proposed to automatically detect fiber orientation on OSB surface with the ultimate aim at optimizing the fiber orientation.

\section{MATERIALS AND METHODS}

The materials selected for the experiment were commercial OSB imported from Germany of $1800 \mathrm{~mm} \times 900 \mathrm{~mm} \times 9 \mathrm{~mm}$. The wood specie is pine (Pinus sylvestris L.). The schematic diagram of the detecting system for the wood strand arrangement or fiber orientation is shown in Figure 1. It consists of a CCD camera (Charge Coupled Device) of the type (Victor KY-F350, Victor JVC), two flood lamps (Toshiba reflector 150WF), a CNC (Computer Numerical Control) working table (Funuc M180 series), and the host computer whose software system was developed and installed by authors. Samples were placed on the 2-dimensional CNC working table and illuminated by two flood lamps from two sides. By adjusting the height and the focus of the digital camera, images of the wood strands were captured under $80 \mathrm{dpi}$ and they were 24-BPP (bit per pixel) gray images. The computer program was written in MatLab Release 12 as code resource. The flow chart of the program is shown in Figure 2.

\section{IMAGE PROCESSING ALGORITHM}

After capturing and inputting the image $g$, Canny edge detector, which was proposed by Canny et al. [1], was operated on it. The Canny method finds edges by looking for local maxima of the gradient of $g$. The gradient is calculated using the derivative of a Gaussian filter. The method uses two thresholds, to detect strong and weak edges, and includes the weak edges in the output only if they are connected to strong edges. It can be described as follows.

(1) Repeat steps (2) till (6) for ascending values of the standard deviation.

(2) Convolve an image $g$ with a Gaussian filter of standard deviation $\sigma$.

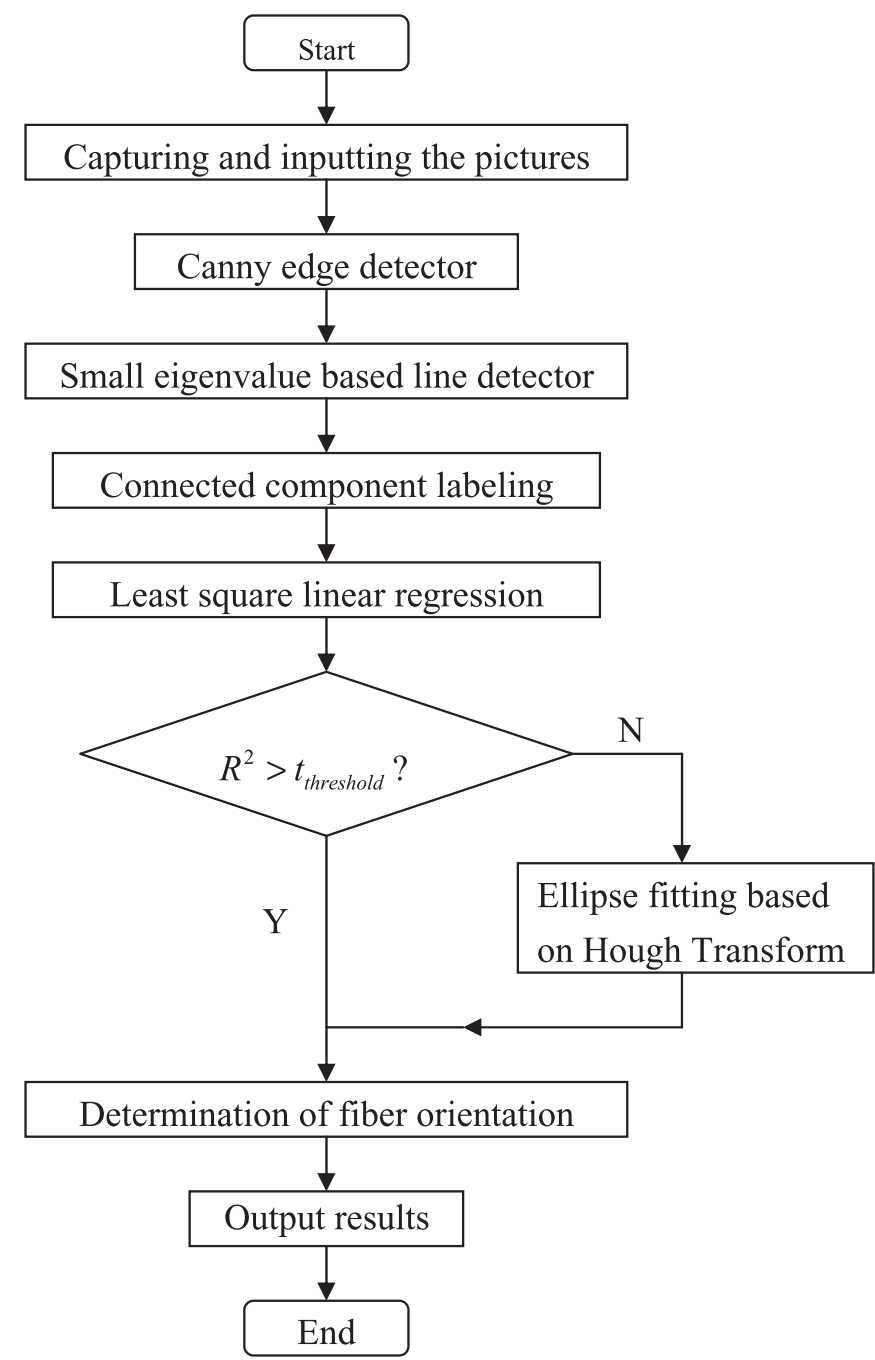

Figure 2. The flow chart of the computer program.

(3) Estimate local edge normal directions $n$ using the following equation for each pixel in the image:

$$
n=\frac{\nabla(G \times g)}{|\nabla(G \times g)|} \text {. }
$$


(4) Find the location of the edges using the following equation for non-maximal suppression:

$$
\frac{\partial^{2}}{\partial n^{2}} G \times g=0 .
$$

(5) Compute the magnitude of the edge using the following equation:

$$
\left|G_{n} \times g\right|=|\nabla(G \times g)| .
$$

(6) Threshold edges in the image with hysteresis (low and high thresholds) to eliminate spurious response.

There are three parameters for Canny edge detector: standard deviation (Sigma), low threshold, and high threshold. Here we determined the three parameters to be $1.2,0.05$, and 0.2 , respectively. After Canny edge detector, the output image called edge image was obtained. Then a novel line detector based on small eigenvalue analysis, which was detailed by Guru et al. [3], was developed and operated. It can be described as follows: Let $g e(M, N)$ be an edge image gotten after canny edge detector. Let $p_{i}(i=1,2, \ldots, n)$ be the edge pixels and $n$ be the total number of edge pixels in $g e$. Let $W$ be a window of size $k \times k$ for some odd integer $k>1$ used for mask processing. The mask window is placed at an edge pixel $p_{i}$. All edge pixels connected to $p_{i}$ and converted by the mask window $W$ is defined to be $F_{i}$, the family set for $p_{i}$. The small eigenvalue $\lambda_{i}$ of $F_{i}$ is computed as follows:

$$
\begin{gathered}
C_{x}=\frac{1}{m} \sum_{i=1}^{m} x_{i} \\
C_{y}=\frac{1}{m} \sum_{i=1}^{m} y_{i} \\
C_{11}=\frac{1}{m} \sum_{i=1}^{m} x_{i}^{2}-c_{x}^{2} \\
C_{22}=\frac{1}{m} \sum_{i}^{2}-c_{y}^{2} \\
C_{12}=C_{21}=\frac{1}{m} \sum_{i=1}^{m} x_{i} y_{i}-c_{x} c_{y} \\
\lambda=\frac{1}{2}\left[c_{11}+c_{22}-\sqrt{\left(c_{11}-c_{22}\right)^{2}+2\left(c_{12}^{2}+c_{21}^{2}\right)}\right]
\end{gathered}
$$

where $x_{i}$ and $y_{i}$ are the $X$ and $Y$ coordinates of the point $p_{i}\left(x_{i}\right.$, $y_{i}$ ) inside $F_{i} ; \mathrm{m}$ is the total number of edge pixels inside $F_{i}$. The mask processing was operated from the bottom left corner to the right up corner. As the successive windows overlap each other, it is common that there are some pixels that are members of more than one family. It is obvious that if an edge pixel only belongs to one family, the pixel is a spurious edge pixel, i.e., it is eliminated as a noise edge pixel. Research results reported that the smaller the eigenvalue of the covariance matrix of connected pixels, the stronger is the evidence that those connected pixels form a linear segment [9]. So if an edge pixel belongs to two or more families, the minimum eigenvalue of families is decided to be the final response value for that pixel. So the proposed transformation function $T: g e$ (an edge image) $\rightarrow g s$ (small eigenvalue image) is defined as follows.

$$
\begin{aligned}
g_{s}(x, y) & =T\left[g_{e}(x, y)\right] \\
& =\left\{\begin{array}{c}
\min \left(\left\{\lambda_{i} \mid g_{e}(x, y) \in F_{i} \forall j=1,2, \ldots, n\right\}\right) \\
\infty
\end{array}\right.
\end{aligned}
$$

if $g_{e}(x, y)$ is an edge pixel and $g_{e}(x, y)$ is not a noise pixel otherwise.

The image called small eigenvalue image is then passed forward to a thresholding operation. If a pixel $g_{e}(x, y)$ is less than a predefined threshold, it is said to be a linear edge pixel and be set a value 1 . If not, then the pixel $g_{e}(x, y)$ is set a value 0 . This binary image is the desired image consisting of only linear edge pixels. This procedure can be expressed as follows:

$$
\begin{aligned}
& g d(x, y)=\tau\left(\left[g_{S}(x, y)\right]\right) \\
& =\left\{\begin{array}{cc}
1 \text { if } \min \left(\left\{\lambda_{i} \mid g_{e}(x, y) \in F_{i} \forall j=1,2, \ldots, n\right\}\right)<t \\
0 \quad \text { otherwise }
\end{array}\right.
\end{aligned}
$$

Then the component connected labeling described by Haralick and Shapiro [4] was used to locate the linear segments on the output image from the line detector based on small eigenvalue analysis. Finally, all linear segments that were detected by component connectedness labeling were passed forward to the linear regression analysis described as follows:

$$
k=\frac{\sum_{i=1}^{n} y_{i} \sum_{i=1}^{n} x_{i}^{2}-\sum_{i=1}^{n} x_{i} \sum_{i=1}^{n} x_{i} y_{i}}{n \sum_{i=1}^{n} x_{i}^{2}-\left(\sum_{i=1}^{n} x_{i}\right)^{2}} .
$$

And the fiber orientation of the individual fiber can be described as follows:

$$
\theta=\operatorname{abs}(\arctan (k)) .
$$

Then the fiber orientation distribution was computed and the orientation of an OSB was defined as the main peak of the distribution.

\section{RESULTS AND DISCUSSION}

The original pictures of the top surface and undersurface are shown in Figures $3 a$ and $4 a$. It can be seen that the flake alignment on the top surface is very clear whereas the flake alignment on the undersurface is blurred because there are many small strands, which contort the edge lines of strands. Nishimura et al. [6] reported that small strands fell when the above multilayer mats were distributed automatically. The final image processed pictures of the top surface and undersurface are shown in Figures $3 b$ and $4 b$. The fiber strands are expressed well using the edge lines detected by line detector on the top surface. However, there are some false edge lines because of 

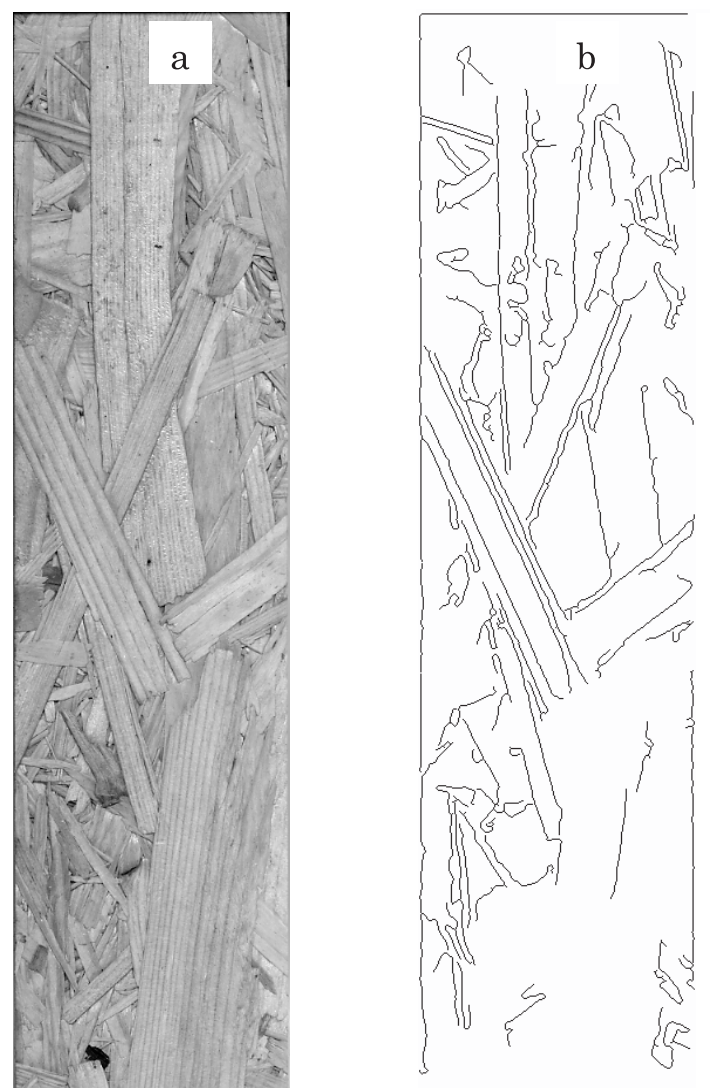

Figure 3. The original picture and the final image processed picture on the top surface. a: Original picture; b: final image processed picture.

the effect of small strands on the edge lines. Canny edge detector and a line detector based on small eigenvalue analysis were used to detect the strands' edge lines. There are three parameters for Canny edge detector (High threshold, Low threshold, and standard deviation) and one parameter for the line detector based on small eigenvalue (threshold $t$ ). The final image processed pictures shown in Figure $4 \mathrm{~b}$ were gotten when three parameters of Canny edge detector were $0.05,0.2$, and 1.5 , respectively, and the threshold parameter of line detector was 0.17625 . This parameter set has a fine performance on the top surface. It is expected that an optimum set of parameters can be achieved to improve the performance of detecting the edge lines after many trials.

The connectedness component labeling was also operated to search for the line objects. Every labeled object was forwarded to least line fitting and/or ellipse fitting to determine the fiber orientation after connectedness component labeling. The rectangle fitting and ellipse fitting analysis based on separating the whole picture into certain numbers of subsets using a window size $M \times N$ were used by Nishimura et al. [6]. However, the captured objects inside a window are often fractures of several components. Since the chance to capture full information, which belongs to a single object inside a window, is very slim, the fiber orientation determined by ellipse or rectangle fitting analysis based on a window is not correct. The method pro-
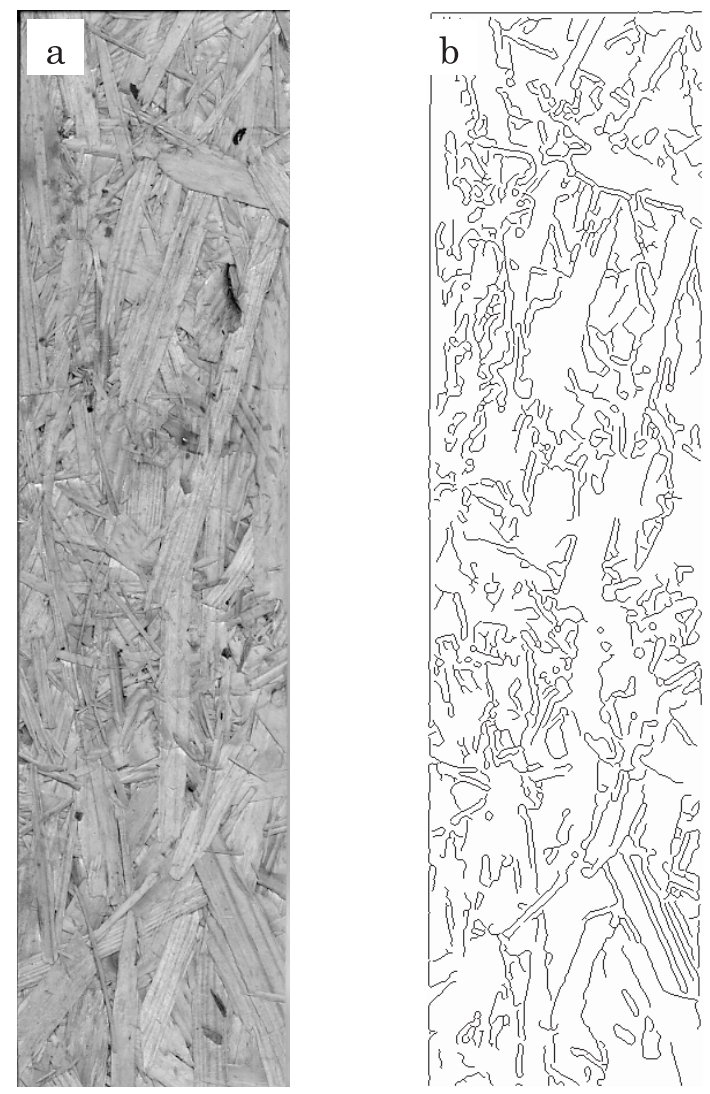

Figure 4. The original picture and the final image processed picture on the undersurface. a: Original picture; $b$ : final image processed picture.

posed in this paper was to try to capture the whole object individually. Then the captured information which belongs to a single object was forwarded to a line fitting and/or ellipse fitting. The histograms of the fiber orientation on the top and under surfaces shown in Figures $3 \mathrm{~b}$ and $4 \mathrm{~b}$ are shown in Figures 5 and 6, respectively. The angle distribution of the top surface is a normal distribution. The central angle of the dominant peak bar is around 23.3 degrees. However, there was no dominant peak, and the frequency distribution of the angles was almost constant in the range 0 to 80 degrees for the undersurface. As described above, the small strands blurred the edge lines of the undersurface because the conveyer movement and vibration worked like a filter to cause the small strands to align random. Most of the small strands fall and collect on the surface of a conveyer, which forms the undersurface of the finished OSB production. Although the big strands have a clear alignment on this surface according to the design product line, the small strands are randomly distributed. This is the reason why there is no dominant bar in the angle distribution. The parameters or other methods should be considered to eliminate the effect of small strands on the edge maps.

A test experiment was conducted to test the robust and effectiveness of the system to determine the fiber orientation. Small samples of $300 \times 75 \times 11 \mathrm{~mm}^{3}$ were cut from a big OSB. The longitudinal direction of the big OSB was defined as a baseline. 


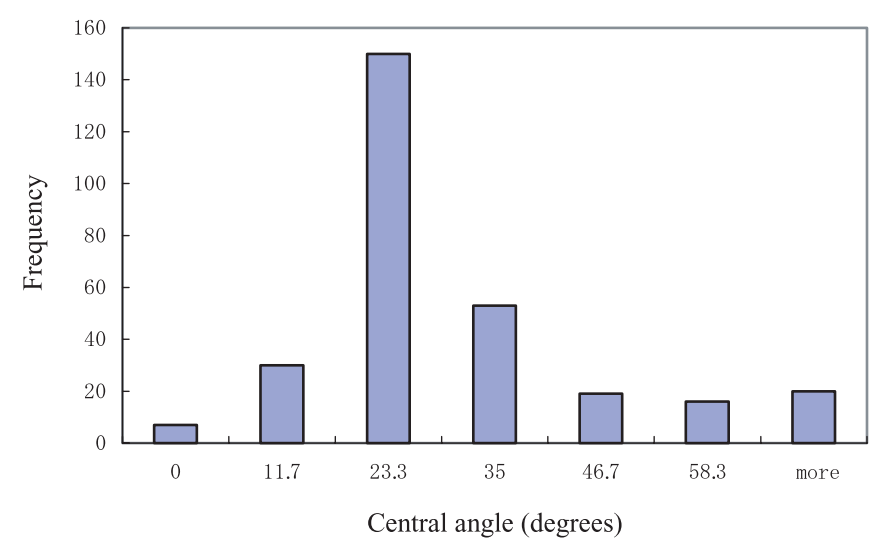

Figure 5. The angle distribution histogram of the top surface. Central angle is the angle at the middle position of a bar in histogram.

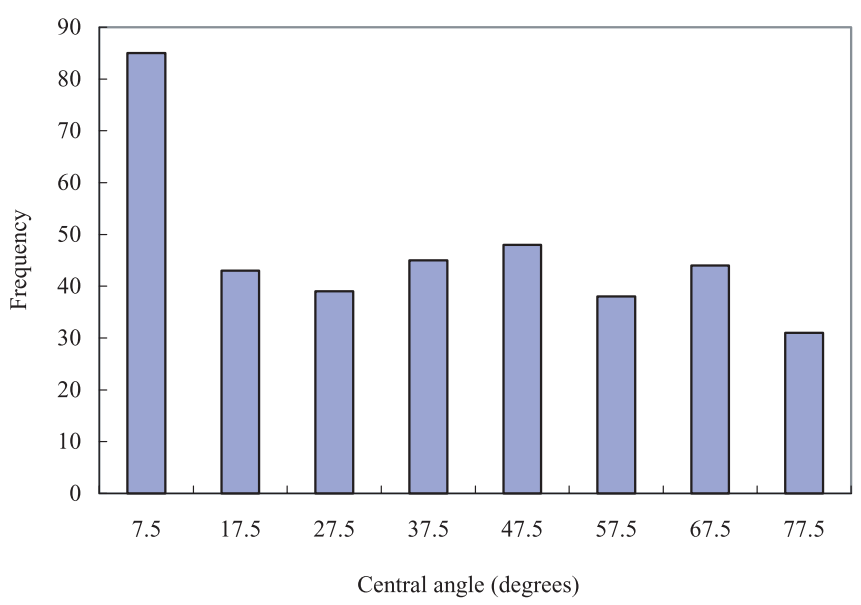

Figure 6. The angle distribution histogramz of the undersurface. Central angle is the angle at the middle position of a bar in histogram.

The acute angle between the longitudinal direction of a small sample and the baseline initially fixed by the authors was varied from 0 to 90 degrees with increment of 15 degrees. Six small samples were obtained for every acute angle. Then the pictures of all small samples were taken and processed by the method developed in this paper. The central and mean angles of histograms for every rotating position are shown in Figure 7. There are two types of angles: mean angle and central angle, which can be used to express the main stream of the flake alignment on the surface of OSB. As shown in Figure 7, it is much better to use the central angle instead of the mean angle to express the fiber orientation. So we propose that when evaluating the quality of OSB, the central angle should be used.

The fiber orientation measured manually and automatically by the method proposed in this paper is shown in Figure 8. It can be observed that the angle measured automatically $(A a)$ is close to the angle measured manually $(A m)$. The ratio of $A a l$ $A m$ is also shown in Figure 8. The ratio varies in the range 0.95 to 1.1 . The two angles are so close that it is reliable to say that the method developed in this paper to determine the fiber orientation is suitable.

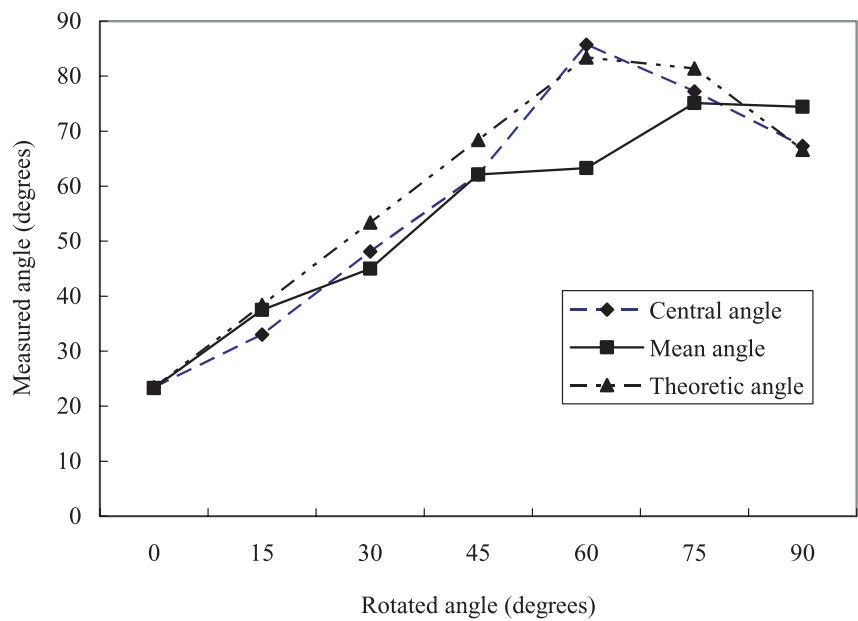

Figure 7. The automatically measured angles at different rotating angles.

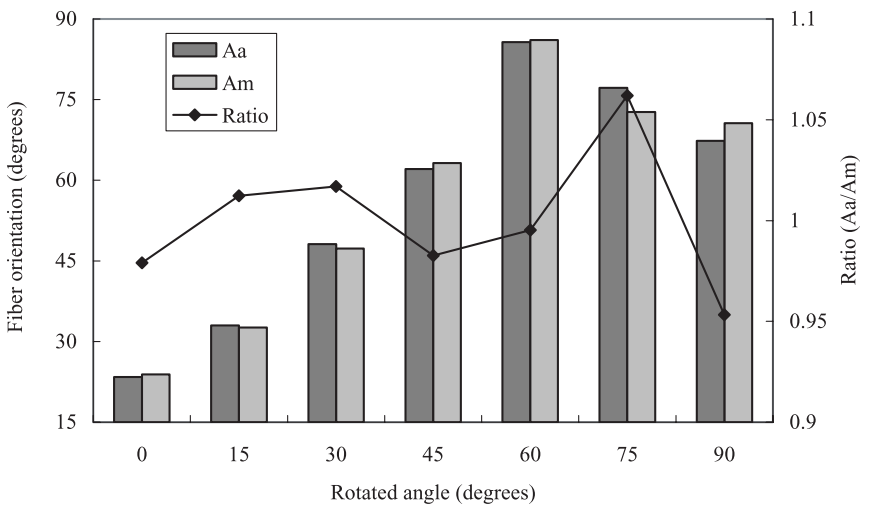

Figure 8. Comparison of automatically and manually measured angles. Aa: the angle measured automatically; Am: the angle measured manually.

The ultimate aim of automatically determining the fiber orientation in OSB is to control the flake alignment in the practical production line so that the quality of OSB can be optimized. Here we just developed an automatic method to determine the fiber orientation on OSB under experimental conditions. But it is expected to apply the algorithm on a flake alignment picture of a distributing mat, which can be taken by a high speed video camera. This topic will be studied later.

\section{CONCLUSIONS}

We developed an automatic system to determine the fiber orientation on the surface of OSB using a novel line detector based on small eigenvalue analysis in conjunction with Canny edge detector. The results have shown that the image analyzing system is effective and suitable to determine the fiber orientation on the top surface of OSB. The measured fiber orientation using the method developed in this paper is very close to that measured manually. The central angle of the histogram is much 
more effective than the mean angle. It is expected that the determined fiber orientation can be used to optimize the strands distribution and the quality control of OSB in the longitudinal direction when the properties in the perpendicular directions are not critical.

However, it is still necessary to do some experiments to optimize the image processing parameters. Also an on-line quality control system will be developed based on on-line monitoring fiber orientation to optimize the flake alignment and the mechanical properties of OSB.

\section{REFERENCES}

[1] Canny A., A computational approach to edge detection, IEEE Trans. PAMI, 8 (1986) 769-698.

[2] Dai C., Steiner R., On horizontal density variation in randomly-formed short fiber wood composite boards, Composites, Part A, 28 (1997) 57-64
[3] Guru D.S., Shekar B.H., Nagabhushan P., A simple and robust line detection algorithm based on small eigenvalue analysis, Pattern Recognition Lett. 25 (2004) 1-13.

[4] Haralick R.M., Shapiro L.G., Computer and robot vision, Vol. 1, University of Washinton, Addison-Wesley Pub. Co. 1992.

[5] Kelly M., Critical review of the relationship between processing parameters and physical properties of particleboard, General Tech. Rep. FPL-10, US FPL, Madison, WI, p. 65.

[6] Nishimura T., Ansell M.P., Ando N., The relationship between the arrangement of wood strands at the surface of OSB and the modulus of rupture determined by image analysis, Wood Sci. Technol. 35 (2001) 555-562.

[7] Pinto I., Pereira H., Usennius A., Analysis of log shape and internal knots in twenty maritime pine (Pinus pinaster Ait.) stems based on visual scanning and computer aided reconstruction, Ann. For. Sci. 60 (2003) 137-144

[8] Suchsland O., Behavior of a particleboard mat during the press cycle, For. Prod. J. 17 (1969) 51-57.

[9] Tsai D.M., Hou H.T., Su H.J., Boundary-based corner detection using eigenvalues of covariance matrices, Pattern Recognition Lett. 20 (1999) 31-40. 